\title{
Electrostatic loading and photoredox-based release of molecular cargo from oligoviologen-crosslinked microparticles
}

Received XX December 2021, Accepted xx ------------ 202x DOI: $10.1039 / \times 0 \times x 00000 x$

\author{
Mark S. Palmquist, Max C. Gruschka, Jovelt M. Dorsainvil, Abigail O. Delawder, Tiana M. Saak, Mary
} K. Danielson, Jonathan C. Barnes*

\begin{abstract}
Although on-demand cargo release has been demonstrated in a wide range of microparticle platforms, many existing methods lack specific loading interactions and/or undergo permanent damage to the microparticle to release the cargo. Here, we report a novel method for electrostatically loading negatively charged molecular cargo in oligoviologen-crosslinked microparticles, wherein the cargo can be released upon activation by visible light. A water-in-oil (W/O) emulsion polymerization method was used to fabricate narrowly dispersed microparticles crosslinked by a dicationic viologen-based dimer and a poly(ethylene glycol) diacrylate. A zinc-tetraphenyl porphyrin photocatalyst was also polymerized into the microparticle and used to photochemically reduce the viologen subunits to their monoradical cations through a visible-lightmediated photoredox mechanism with triethanolamine (TEOA) as a sacrificial reductant. The microparticles were characterized by microscopy methods revealing uniform, spherical microparticles $481 \pm 20.9 \mathrm{~nm}$ in diameter. Negatively charged molecular cargo (methyl orange, $\mathrm{MO}$ ) was electrostatically loaded into the microparticles through counteranion metathesis. Upon irradiation with blue $(450 \mathrm{~nm})$ light, the photo-reduced viologen crosslinker subunits lose positive charges, resulting in release of the anionic MO cargo. Controlled release of the dye, as tracked by absorption spectroscopy, was observed over time, yielding release of up to $40 \%$ of the cargo in $2 \mathrm{~d}$ and $60 \%$ in $5 \mathrm{~d}$ in single dynamic dialysis experiment. However, full release of cargo was achieved upon transferring the microparticles to a fresh TEOA solution after the initial $5 \mathrm{~d}$ period.
\end{abstract}

\section{Introduction}

With a multi-billion dollar global market $^{1}$ and increasing demand for versatile applications, polymer-based microparticles have been investigated extensively. Although the main focus has been on fabricating microparticles to yield uniform shapes and sizes, ${ }^{2-4}$ many researchers have turned their attention to developing next-generation microparticles. Specifically, microparticles that can respond to external environmental cues are of interest because they have served as platforms for cargo release in roles such as targeted drug delivery ${ }^{5-7}$ and self-healing materials. ${ }^{8}$

On-demand cargo release from microparticles has been demonstrated by various methods including chemical activation using self-immolative capsules $^{9}$ and mechanical activation through damage to polymer shell walls. ${ }^{10,11}$ These mechanisms display efficient time-specific release of cargo, however, chemically activated microparticles often lack precise temporal control, whereas physical fracturing of microparticles usually causes lasting damage to the delivery vehicle. Microparticles designed for spatiotemporal-controlled release without permanent damage to the platform are therefore desirable.

Department of Chemistry, Washington University in St. Louis, One Brookings Drive, St. Louis, MO 63130, USA. E-mail: jcbarnes@wustl.edu

Electronic Supplementary Information (ESI) available: See DOI: 10.1039/x0xx00000x
Photo-stimulated microparticles are thus well suited as an attractive option that blends such characteristics for use in drug-release ${ }^{12,13}$ and self-healing ${ }^{14}$ applications, among others.

As a dicationic electron-acceptor, viologens ${ }^{15}, 16$ are a promising option to facilitate electrostatic binding and subsequent release of negatively charged molecular cargo upon reduction of the viologen subunits $\left(\mathrm{V}^{2+}\right.$ to $\left.\mathrm{V}^{\bullet+}\right)$. Viologens, namely, $N, N^{\prime}$-dialkyl-4,4'-bipyridiniums, are highly redox-active compounds that are well-studied with reduction potentials that are compatible with many different photocatalysts. Previously, we investigated the effects of oligoviologen-based crosslinkers that were chemically ${ }^{17}$ and photochemically ${ }^{18,} 19$ reduced in situ to actuate and spatiotemporally photopattern ${ }^{20}$ gels. To achieve the photoreduction initially, ruthenium tris-bipyridine dichloride $\left(\left[\mathrm{Ru}(\mathrm{bpy})_{3}\right] \mathrm{Cl}_{2}\right)$ and a sacrificial reductant (triethanolamine, TEOA) were swelled into the gels prior to irradiation with $450 \mathrm{~nm}$ light. Recently, we transitioned to a zinc tetraphenyl porphyrin as an integrated photocatalyst to expand the absorption wavelength profile of the gels in the visible region, wherein actuation was demonstrated using both blue and red light. ${ }^{21}$ The mechanism of contraction resulted, in part, from intramolecular recognition between viologen monoradical cation subunits in the oligomeric crosslinker, driven by favourable radical-radical spin pairing. ${ }^{22,} 23$ The decrease in electrostatic repulsion, as well as mass loss in the form of expelled anions, also contributed to gel contractions. 


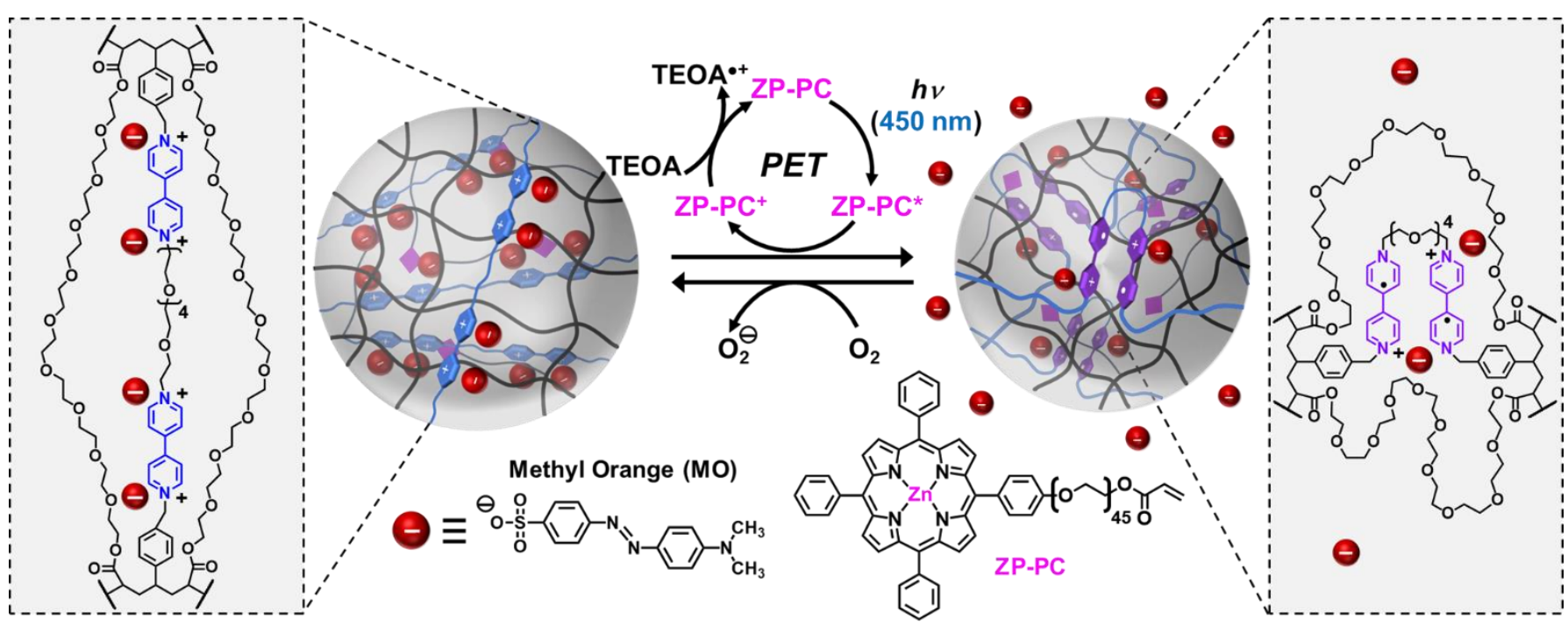

Figure 1-Photoinduced electron transfer (PET) within oligoviologen-crosslinked microparticles containing zinc porphyrin photocatalysts results in a reduction and reoxidation mechanism that facilitates release of electrostatically-bound dye molecules in response to blue light (450 $\mathrm{nm}$ ).

The $\mathrm{V}^{2+}$ to $\mathrm{V}^{\bullet+}$ transition has been leveraged in various applications $22,24,25$ because of its reversibility, where $\mathrm{V}^{\bullet+}$ quickly oxidizes back to $\mathrm{V}^{2+}$, typically in seconds, upon exposure to $\mathrm{O}_{2}$, the latter of which is converted (Fig. 1) to superoxide anion $\left(\mathrm{O}_{2}{ }^{-}\right.$ ). ${ }^{26}$ The viologen reduction step in this case is therefore critical for applications in controlled release as each viologen dication loses half its positive charges, allowing the charge-balancing counteranions to diffuse away. ${ }^{17}$ In the presence of $\mathrm{O}_{2}$, this photoredox process is dynamic and can continuously trigger the release of counteranions from all the oligoviologen subunits. Traditionally, inorganic anions $\left(\mathrm{Cl}^{-}, \mathrm{PF}_{6}{ }^{-}\right.$, etc.) have been used to facilitate viologen solubility ${ }^{16}$ and isolation through facile exchange and precipitation methods. Instead, we now propose to use anions that possess greater functionality. Specifically, molecular cargos that may be installed through anion metathesis exchange, thus setting the stage for a method of electrostatically binding functional anions that can be released on-demand through a novel photoreduction mechanism.

Herein, we describe a photo-responsive microparticle (Fig. 1) in which negatively charged molecular cargo was electrostatically loaded and subsequently released oncommand through a photoinduced electron transfer (PET) from integrated zinc porphyrin photocatalysts to oligoviologen-based crosslinkers. Using a water-in-oil (W/O) emulsion polymerization technique, microparticles were synthesized by combining an oligoviologen dimer with a poly(ethylene glycol) diacrylate (PEGDA) as co-crosslinkers. The resultant microparticles were characterized by elemental analysis and optical and electron microscopy methods, demonstrating narrowly dispersed particles $481 \pm 20.9 \mathrm{~nm}$ in diameter. To assess the release kinetics, negatively charged methyl orange (MO) was chosen as the molecular cargo since its controlled release could be readily monitored by absorption spectroscopy over the span of several days. As control experiments, the release of MO was compared across microparticle batches (i) with and without light irradiation, (ii) without an integrated photocatalyst, or (iii) having no oligoviologen crosslinker present. The results of this investigation demonstrate that optimal cargo release is achieved when all components - the oligoviologen crosslinker, integrated photocatalyst, and blue light - were used in concert.

\section{Results and Discussion}

\section{Synthesis of viologen-crosslinker and photocatalysts}

To create a microparticle capable of electrostatic loading and release, we synthesized a redox-responsive crosslinker consisting of two viologen subunits linked by hexaethylene glycol (HEG) and capped with polymerizable styrene groups. The dimer was chosen because it can be synthesized in only a few steps (vide infra) and is very water soluble; a critical attribute necessary to synthesize narrowly dispersed microparticles via an emulsion polymerization. Briefly, 1 equiv of ditosylated hexaethylene glycol (DiTos HEG) and 20 equiv of 4,4'-bipyridine were heated to $200{ }^{\circ} \mathrm{C}$ for $15 \mathrm{~min}$ in MeCN (25 $\mathrm{mg} \cdot \mathrm{mL}^{-1}$ DiTos HEG) in a microwave reactor at $150 \mathrm{~W}$ (300 psi pressure limit). The product solution was purified via selective precipitation from $\mathrm{MeCN}$ using $\mathrm{Et}_{2} \mathrm{O}$ and centrifugation to isolate the pseudo-viologen $\mathbf{2 V} \cdot 2 \mathrm{OTs}$ in $92 \%$ yield. To complete the crosslinker synthesis, one equiv of $\mathbf{2 V} \cdot 2 \mathrm{OT}$ s and 40 equiv of 4-vinylbenzyl chloride were dissolved in DMF (dry, $50 \mathrm{mg} \cdot \mathrm{mL}^{-1}$ $2 \mathrm{~V} \cdot 2 \mathrm{OTs}$ ) and the solution was heated to $60^{\circ} \mathrm{C}$ for $3 \mathrm{~d}$. Upon completion of the reaction, the solution was purified in the same manner as $\mathbf{2} \mathbf{V} \cdot \mathbf{2 O}$ Ts. The resultant solid was pelleted via centrifugation, redissolved in $\mathrm{MeCN}$, and precipitated once more in $\mathrm{Et}_{2} \mathrm{O}$, followed by another round of centrifugation. This purification process was repeated a total of three times to ensure complete removal of excess 4-vinylbenzyl chloride. The compound was converted to a $\mathrm{PF}_{6}{ }^{-}$salt using $\mathrm{NH}_{4} \mathrm{PF}_{6}$, and back to the water-soluble $\mathrm{Cl}^{-}$salt using TBACl to yield $\mathbf{2} \mathbf{V}-\mathrm{St} \cdot 4 \mathrm{Cl}$ in an $88 \%$ yield. For additional details about the synthesis and characterization of the crosslinker, see Section B. Synthetic Protocols in the Supporting Information (SI).

Next, a polymerizable photocatalyst was synthesized to imbue the microparticle with the ability to absorb visible light and reduce the viologen-based crosslinker through PET. To accomplish this, we modified a previous synthesis ${ }^{21}$ of an 
a

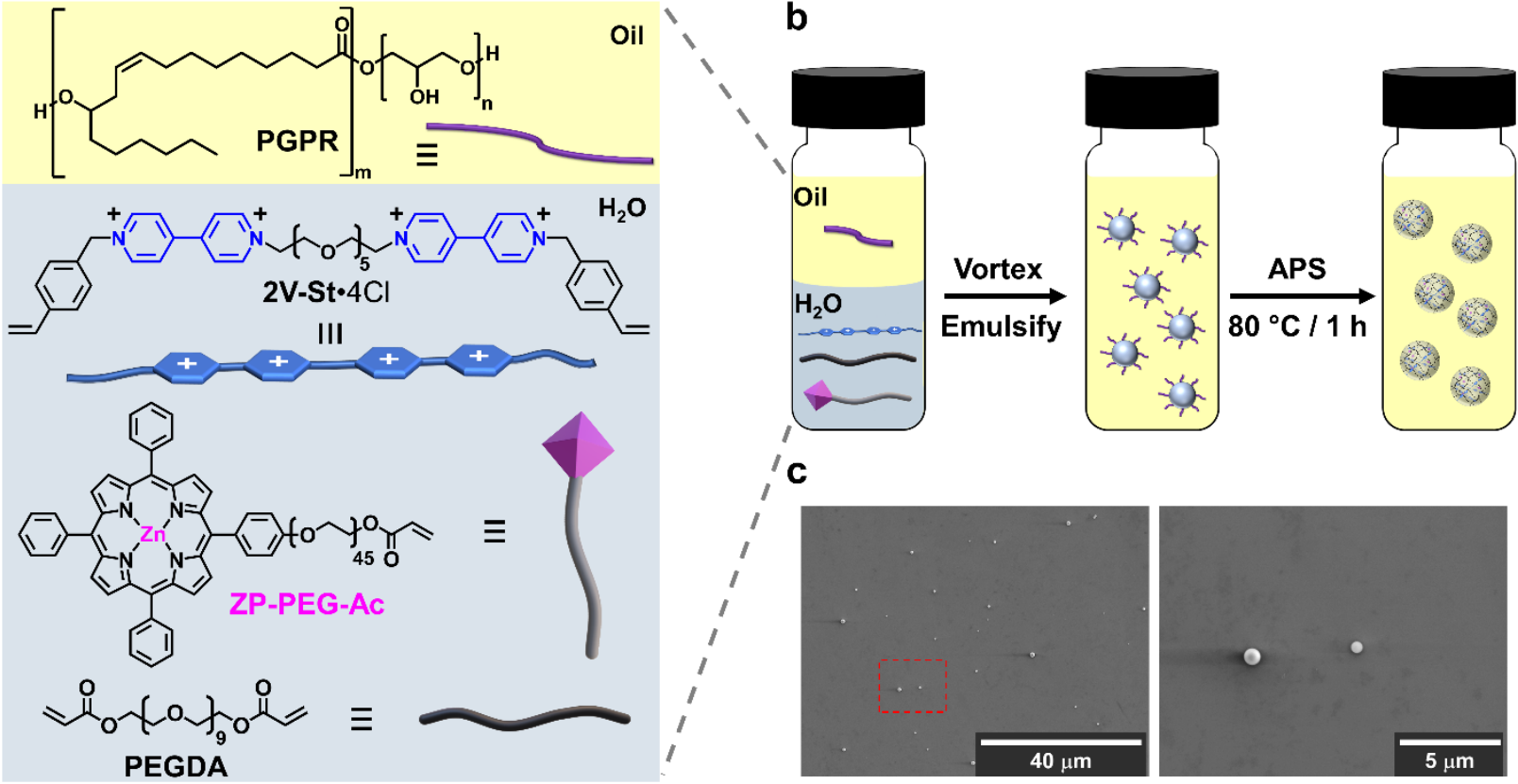

Figure 2 - a) Microparticle ingredients in their respective oil or water phase. b) Microparticle emulsion and polymerization scheme initiated by ammonium persulfate (APS). c) (Left) Scanning Electron Microscopy (SEM) image of as-synthesized microparticles (2,000x magnification), (Right) SEM image (10,000x magnification) of red box region shown in the 2000x image.

acrylate-functionalized zinc tetraphenyl porphyrin with a PEG spacer $\left(\mathrm{MW}=2000 \mathrm{~g} \cdot \mathrm{mol}^{-1}\right)$ to facilitate water solubility. Hydroxy tetraphenyl porphyrin (TPP-OH) was synthesized by heating 3 equiv benzaldehyde, 1 equiv hydroxybenzaldehyde, and 4 equiv pyrrole in propionic acid at reflux for $4 \mathrm{~h}$. The solution was cooled to $-40{ }^{\circ} \mathrm{C}$ and the precipitate was collected and purified by silica column chromatography in a $5 \%$ yield. TPP-OH was then linked with a monotosylated PEG chain by heating 2 equiv TPP-OH, 1 equiv monotosylated PEG (MW 2000), and 20 equiv $\mathrm{K}_{2} \mathrm{CO}_{3}$ in DMF (dry, $15 \mathrm{mg} \cdot \mathrm{mL}^{-1}$ PEG-OTs) at $80{ }^{\circ} \mathrm{C}$ overnight. The product solution was purified by silica column chromatography to isolate the desired PEG-linked porphyrin (TPP-PEG-OH) in $89 \%$ yield and to recover excess TPP-OH. An acrylate handle was added by stirring 1 equiv TPPPEG-OH, 4 equiv $\mathrm{Et}_{3} \mathrm{~N}$, and 3 equiv acryloyl chloride in $\mathrm{CH}_{2} \mathrm{Cl}_{2}$ (dry, $20 \mathrm{mg} \cdot \mathrm{mL}^{-1}$ TPP-PEG-OH) at RT overnight. The product was purified by washing the organic phase with saturated $\mathrm{NaHCO}_{3}$ and brine solutions to obtain the acrylate functionalized porphyrin (TPP-PEG-Ac) in a $95 \%$ yield. The porphyrin photocatalyst synthesis was completed by stirring 1 equiv of TPP-PEG-Ac with 10 equiv $\mathrm{Zn}(\mathrm{OAc})_{2}$ in a 70:30 $\mathrm{CHCl}_{3}: \mathrm{MeOH}$ mixture (10 $\mathrm{mg} \cdot \mathrm{mL}^{-1}$ TPP-PEG-Ac) at RT overnight. The product was purified by washing the organic phase with $\mathrm{H}_{2} \mathrm{O}$ to produce the zinc porphyrin PEG photocatalyst, ZP-PEG-Ac, in a $97 \%$ yield.

\section{Fabrication of photoredox-enabled microparticles}

The microparticles were synthesized (Fig. 2) using a W/O emulsion polymerization technique, where the aqueous phase contained 2V-St•4Cl, ZP-PEG-Ac, PEGDA co-crosslinker ( $\mathrm{MW}=$ $\left.575 \mathrm{~g} \cdot \mathrm{mol}^{-1}\right)$, and ammonium persulfate (APS) as the radical initiator (see Tables S1-2 for exact reagent stoichiometry). Water solubility was found to be a critical characteristic for monodisperse, uniform microparticle formation. With greater solubility of the aqueous components, each emulsified droplet could contain crosslinker and photocatalyst in greater density. Along these lines, the HEG linker between the viologen subunits of the crosslinker was needed so more $2 \mathrm{~V}$-St $\bullet 4 \mathrm{Cl}$ could be incorporated into the microparticles relative to PEGDA, which in turn increased the loading capacity. However, the PEGDA cocrosslinker was added to achieve better mass yields for each microparticle batch. Through several screens, a vegetable oil continuous phase supported by a polyglycerol polyricinoleate (PGPR) emulsifier was chosen based on emulsions commonly used in industry. ${ }^{27-30}$ Vegetable oil containing PGPR (Fig. 2a) was added to a separate vial such that the oil:water ratio (wt:wt) was 9:1. The aqueous mixture was transferred by micropipette to the vial containing the oil and emulsifier. The combined mixture was vortexed (Fig. 2b) for one minute yielding good emulsion stability and uniform water droplet dispersion. The emulsion mixture was placed in an $80^{\circ} \mathrm{C}$ oven for $1 \mathrm{~h}$ to form microparticles. During heating, the APS initiator polymerized the co-crosslinkers and photocatalyst from the inside of each water droplet. PGPR, an emulsifier used commonly in the chocolatiering industry, ${ }^{31}$ was chosen specifically for its performance in stabilizing emulsions at higher temperatures, where other emulsifiers screened in this investigation failed to maintain water droplet separation. After $1 \mathrm{~h}$, the microparticles suspended within vegetable oil were washed with hexanes to remove the residual oil, followed by transferring to centrifuge tubes to collect the microparticles by centrifugation. The microparticles were then resuspended and washed with $\mathrm{MeOH}$ by centrifuging to remove any remaining unpolymerized material, yielding viologen-based microparticles (MP-1) with integrated zinc porphyrin photocatalyst in $61 \%$ mass yield. To calculate the yield for each batch, the microparticles were taken up in $\mathrm{MeOH}$ to an exact volume of $10 \mathrm{~mL}$ and mixed well to generate a thorough suspension. A $500 \mu \mathrm{L}$ aliquot was taken and transferred to a pre-tared vial. The vial was dried under 
vacuum and the aliquot mass was noted. The total yield for the batch was then calculated by multiplying the aliquot mass by a factor of 20.

\section{Evaluation of viologen and photocatalyst integration}

The microparticles were evaluated to ensure viologen and photocatalyst integration. An aliquot of microparticles were swelled in $2 \mathrm{~mL}$ of $3 \mathrm{mM}$ TEOA in $\mathrm{H}_{2} \mathrm{O}$. The solution was degassed, transferred to an inert $\mathrm{N}_{2}$ environment, and irradiated for $60 \mathrm{~min}$ with $450 \mathrm{~nm}$ light. Before and after pictures (Fig. S4) were taken and the darkened colour of the suspension indicated that viologen units within the microparticles were reduced upon irradiation of the integrated photocatalyst. Chemical reduction of viologen subunits in the microparticles was also tested with addition of $0.1 \mathrm{M}$ sodium dithionite $\left(\mathrm{Na}_{2} \mathrm{~S}_{2} \mathrm{O}_{4}\right)$ in $\mathrm{H}_{2} \mathrm{O}$ (Fig. S5). The microparticles changed colour to a deep purple indicative of viologen reduction (i.e., $\mathrm{V}^{2+}$ to $\mathrm{V}^{\bullet+}$ ). The reduced microparticles were filtered to prove the purple colour was not due to unpolymerized $\mathbf{2} \mathbf{V}-\mathbf{S t} \bullet 4 \mathrm{Cl}$ in solution. Both microparticle variants were further qualitatively evaluated under an optical microscope (Fig. S6) to show uniformly spherical individual microparticles with a monomodal size distribution (vide infra). The microparticle aliquots were observed before and after addition of $\mathrm{Na}_{2} \mathrm{~S}_{2} \mathrm{O}_{4}$ to visualize microparticle reduction (Fig. S6).

\section{Electrostatic loading of methyl orange dye}

To demonstrate oligoviologens can be loaded with organic anions, methyl orange (MO) dye was selected as the molecular

a

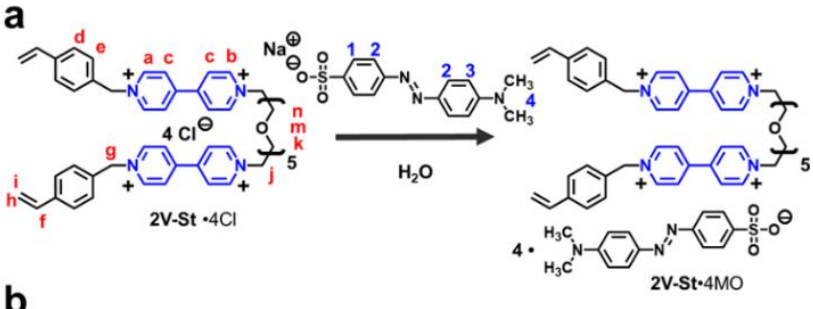

b

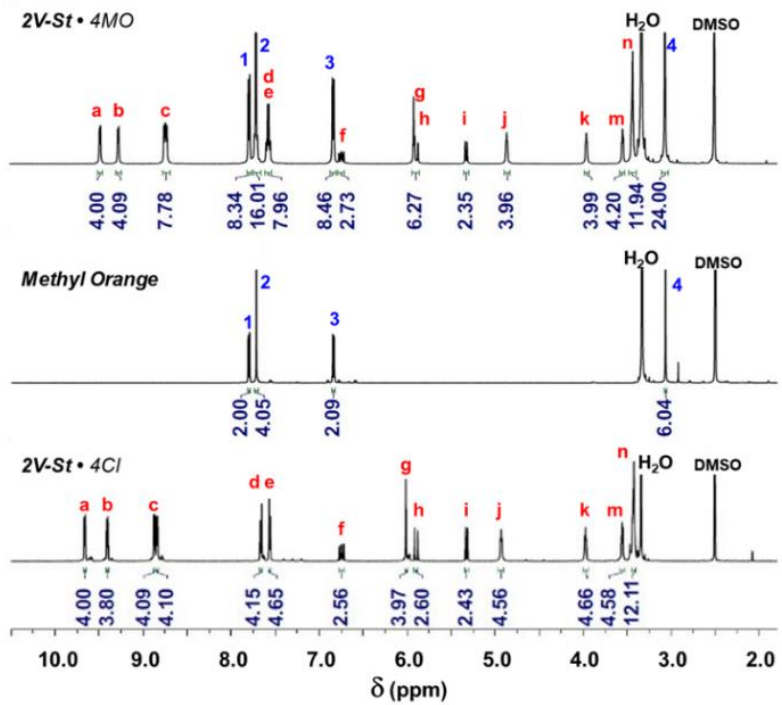

Figure $\mathbf{3}-\mathrm{a}$ ) Loading of $\mathrm{MO}$ dye onto $\mathbf{2 V}$-St $\bullet 4 \mathrm{Cl}$ crosslinker. b) ${ }^{1 \mathrm{H}} \mathrm{NMR}$ of assynthesized dimer crosslinker, methyl orange dye, and loaded small molecule after metathesis. ${ }^{1} \mathrm{H} \mathrm{NMR},\left(\mathrm{CD}_{3}\right)_{2} \mathrm{SO}, 500 \mathrm{MHz}$.

\begin{tabular}{|c|c|c|}
\hline Sample/Control & $\begin{array}{c}\text { Viologen } \\
\text { Crosslinker }\end{array}$ & Photocatalyst \\
\hline MP-1 & $\mathrm{Y}$ & $\mathrm{Y}(\mathrm{Zn})$ \\
\hline MP-2 & $\mathrm{Y}$ & $\mathrm{Y}(\mathrm{Ru})$ \\
\hline MP-3 & $\mathrm{Y}$ & $\mathrm{N}$ \\
\hline MP-4 & N (PEGDA only) & $\mathrm{Y}$ \\
\hline MP-1_No-hv & $\mathrm{Y}$ & $\mathrm{Y}$ \\
\hline
\end{tabular}

Table 1 - Reagents included in microparticle and control batches

cargo (Fig. 3a) to undergo anion metathesis with the chloride salt of the oligoviologen crosslinker. To test loading, 1 equiv of $2 \mathrm{~V}-\mathrm{St} \bullet 4 \mathrm{Cl}$ was dissolved in minimal $\mathrm{H}_{2} \mathrm{O}$ in a centrifuge tube, and a separate solution containing 10 equiv of $\mathrm{MO}$ dissolved in minimal $\mathrm{H}_{2} \mathrm{O}$ was added, leading to precipitation of an orange solid. The solid was pelleted by centrifuge, the supernatant was discarded, and the pellet was washed repeatedly by centrifuge with $\mathrm{H}_{2} \mathrm{O}$ until the aqueous supernatant layer was very faint in colour, yielding $\mathbf{2 V}$-St $\bullet 4 \mathrm{MO}$ in $70 \%$ yield $(75 \mathrm{mg}$ ). Proton nuclear magnetic resonance ( ${ }^{1} \mathrm{H} N \mathrm{NMR}$ ) was used (Fig. $3 \mathrm{~b}$ ) to characterize the product, 2V-St・4MO, 2V-St•4Cl, and MO. A quick comparison of the integration of viologen proton resonance ' $a$ ' in Fig. $3 \mathrm{~b}$ to the MO proton resonance ' 4 ' shows a 4:24 ratio that is expected for introducing four $\mathrm{MO}$ anions for every oligoviologen crosslinker.

Following the successful proof-of-concept for anion exchange at the molecular level, MO loading in microparticles was accomplished post-polymerization (Fig. 4a). MP-1, as well as control microparticle batches MP-3, MP-4, and MP-1_No-hv, (Table 1), were transferred to separate 8-dram scintillation vials as suspensions in $\mathrm{MeOH}$. Excess $\mathrm{MO}$ was added to a separate vial and dissolved in a 50:50 mix of $\mathrm{MeOH}: \mathrm{H}_{2} \mathrm{O}$. The $\mathrm{MO}$ solution was combined with each microparticle solution, and the resulting suspensions were placed on a shaker table to ensure complete counteranion exchange. The microparticles were washed with $50: 50 \mathrm{MeOH}: \mathrm{H}_{2} \mathrm{O}$ several times by centrifuge, and the microparticles were taken up in $\mathrm{MeOH}$ to an exact volume of $10 \mathrm{~mL}$ before being mixed well to afford a homogeneous suspension.

The mass yield after loading MO was calculated using an aliquot and tared vials as described earlier. The loaded microparticles were qualitatively checked under an optical microscope and compared to the as-synthesized precursor microparticles (Figs. 4b, S8). The MO-loaded MP-1 microparticles were observed to aggregate together much more than the as-synthesized precursor, but the spherical morphology was conserved (Fig. 4b). To demonstrate the microparticle platform could be constructed with different photocatalysts, we also synthesized an acrylate-functionalized ruthenium photocatalyst by modifying previously reported syntheses. ${ }^{32-35}$ Microparticles were fabricated by the same method as the zinc porphyrin-based microparticles. Assynthesized $\mathrm{Ru}(\mathrm{bpy})_{3}$ microparticles were uniform in size and distribution on the same scale as MP-1. However, upon MO loading, the $\mathrm{Ru}(\mathrm{bpy})_{3}$ microparticles (MP-2) were clumped and aggregated into intractable clusters when loaded with $\mathrm{MO}$ and could not be analysed and measured by microscopy or other characterization efforts (Fig. S8, top). For this reason, only MP- 
a
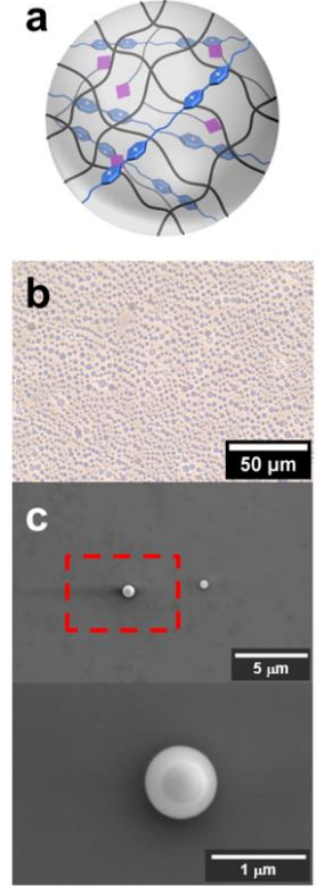

Methyl Orange $\Theta \equiv$ MO

$\mathrm{MeOH} / \mathrm{H}_{2} \mathrm{O}$
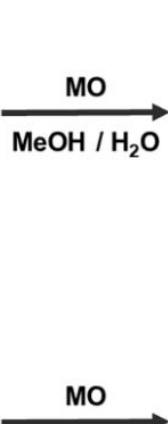

$\mathrm{MeOH} / \mathrm{H}_{2} \mathrm{O}$
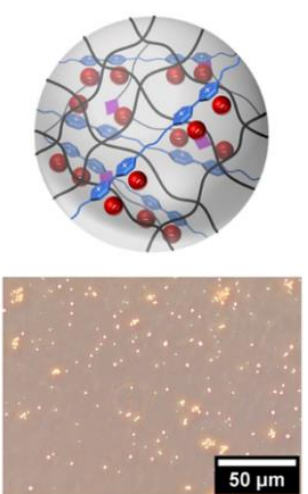

Figure 4 - a) Illustration showing counteranion metathesis as a method for loading methyl orange (MO) into MP-1. b) Optical microscopy images of microparticles before (left) and after (right) loading of MO. c) Scanning electron microscopy (SEM) images of microparticles before (left) and after (right) loading of $\mathrm{MO}$. 10,000x magnification (top) with a zoomed image of the red box at 65,000x magnification (bottom).

1 microparticles were taken forward for further characterization. To quantitatively establish loading capacity of the microparticles, elemental analysis was used to calculate nitrogen $(\mathrm{N})$ content of both as-synthesized and $\mathrm{MO}$-loaded microparticles. Theoretically, the $\mathrm{N}$ content (wt/wt\%) was calculated to be 3.78 and $8.67 \%$ respectively. By elemental analysis, $\mathrm{N}$ content was determined to be 3.07 and $8.26 \%$ respectively. Comparing the as-synthesized $\mathrm{N}$ content values, we observed that $81.3 \%$ of $\mathrm{N}$-containing precursors were successfully polymerized into the microparticles. Using this percentage and assuming PEGDA and APS were polymerized into the microparticles by the same fraction, the cargo loading capacity was calculated (Fig S9) to be $2.625 \mu$ mols cargo per $\mathrm{mg}$ of unloaded microparticles. The loaded $\mathrm{N}$ content was then compared to the theoretical value. Because the expected and theoretical $\mathrm{N}$ values agreed so closely, the MO loading of the particles was deemed nearly quantitative, which is consistent with the efficient solution-phase anion exchange observed for the small-molecule oligoviologen crosslinker (Fig. 3).

\section{Characterization of microparticle size pre- and post-loading}

Scanning electron microscopy (SEM) was used (Fig. 4c) to evaluate the size and morphology of the microparticles. SEM images of the as-synthesized and MO-loaded microparticles were recorded at 2,000,6,500, 10,000, 20,000, and 65,000x magnification (Fig. S10a-e) and showed microparticles that were uniformly spherical in shape and possessing a monomodal size distribution $(481 \pm 20.9 \mathrm{~nm})$. At the highest magnifications (Fig. 4c, bottom), we observed a corona morphology in the microparticles, where the interior and exterior appear to have

different densities. We hypothesize this may be due to the inside-out polymerization of the crosslinker ingredients within the emulsified droplets of $\mathrm{H}_{2} \mathrm{O}$. By measuring the respective diameters from the SEM images (Fig. S11), the outer sphere corona volume was found to be $77.6 \pm 5.85 \%$ of the total microparticle volume. Despite this corona morphology, the MO was still capable of loading into the core to produce quantitative loading (vide infra). To calculate the size and distribution of the microparticles before and after cargo loading, three SEM images at 2,000x magnification were analysed by ImageJ in conjunction with dynamic light scattering (DLS, Figs. S12-13). The SEM-generated scalebar was used to equate ImageJ pixels to a known distance. ImageJ measurement programming was used to compute diameters for all microparticles in each image (Table S5), resulting in the as-synthesized microparticles being $481 \pm 20.9 \mathrm{~nm}$ in diameter and the loaded microparticles measuring $785 \pm 18.2 \mathrm{~nm}$ in diameter. By DLS (Figs. S12, S13, Table S6), the as-synthesized microparticles measured $975.1 \pm$ $93.8 \mathrm{~nm}$ and the MO-loaded microparticles measured $2688.2 \pm$ 227.2. Although DLS gave sizes more than twice that shown by SEM, each method demonstrated microparticle swelling after loading with the bulkier MO counteranion, which suggested successful preparation of electrostatically loaded microparticles.

DLS was also used to track the size of MP-1 in response to chemical reduction via $\mathrm{Na}_{2} \mathrm{~S}_{2} \mathrm{O}_{4}$ solution. A $1 \mathrm{M}$ solution of the reductant was added to the as-synthesized microparticles in a DLS cuvette, followed by measuring the size of the microparticles at $1,3,5,15$, and 30 min timepoints after mixing. The microparticles quickly exhibited a reduction in size to 471.9 $\pm 79.1 \mathrm{~nm}$ after $1 \mathrm{~min}$ before returning to their initial size and swelling slightly over the remaining timepoints. We speculate the swelling after the initial contraction was due to a combination of two factors. Counteranion metathesis between the smaller chloride anion $\left(\mathrm{Cl}^{-}\right)$and the larger dithionite dianion $\left(\mathrm{S}_{2} \mathrm{O}_{4}{ }^{2-}\right)$ that was present in excess in solution results in swelling of the microparticle, even as the viologen subunits were reduced. Additionally, the swelling of the microparticles may have also occurred as the result of ambient $\mathrm{O}_{2}$ that re-oxidized the reduced viologen subunits within the microparticles. In our previous reports, the sustained physical contraction of a viologen-based gel network was a critical characteristic of reduction in an inert environment, combining loss of $\mathrm{H}_{2} \mathrm{O}$ and counteranions with intramolecular self-assembly of the polyviologen subunits. ${ }^{17-19}$ However, in the heavily cocrosslinked microparticles described here, the contraction was only observed briefly in the presence of $\mathrm{O}_{2}$, and the microparticle size returned quickly to its pre-reduced size and even swelled slightly larger. Because the main goal of developing the photoredox-based microparticles is to enable electrostatic loading and light-induced release, the lack of controlled contraction in the extensively crosslinked microparticles is of no consequence.

\section{Controlled release of methyl orange from the microparticles}

To measure the release kinetics of $\mathrm{MO}$ from the microparticles, MO-loaded samples were analysed in triplicate 

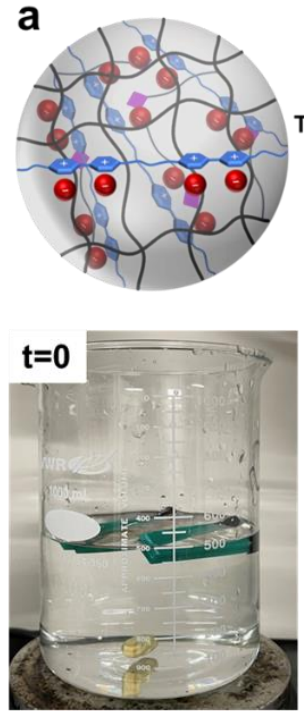

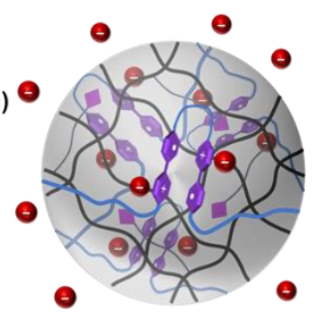

Q
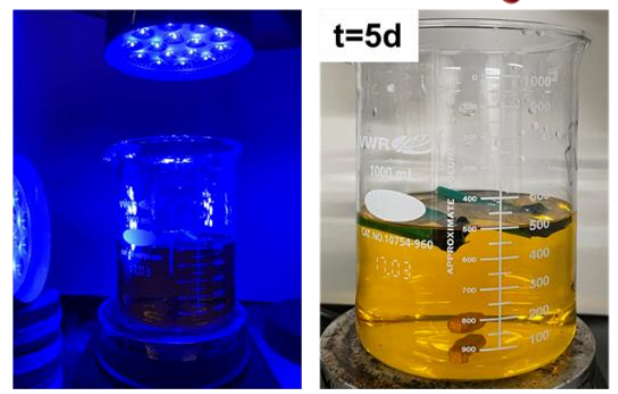

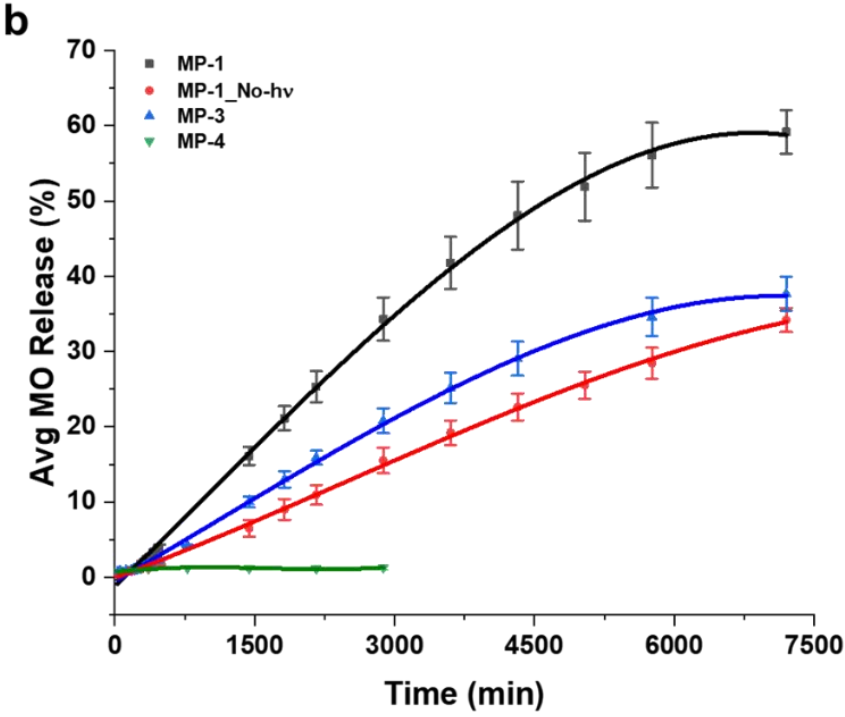

Figure 5 - Photoinitiated release of methyl orange over time. a) MO release scheme and pictured setup of dialysis tubing containing loaded microparticles before, during, and after irradiation with $450 \mathrm{~nm}$ light. b) Percent release of MO from microparticle samples and controls over $5 \mathrm{~d}$.

in conjunction with the control microparticle batches MP-3, MP-4, and MP-1_No-hv, where oligoviologen, photocatalyst, and exposure to light were removed from the microparticle composition or treatment (Table 1). Aliquots of microparticles (10 mg) were transferred to dialysis tubing (SpectraPor 7 RC, 45 $\mathrm{mm}$ width, $8 \mathrm{kD}$ cut-off) and soaked in $\mathrm{H}_{2} \mathrm{O}$ for $36 \mathrm{~h}$ to dialyze any non-electrostatically bound dye. Next, the microparticles were transferred to beakers containing $3 \mathrm{mM}$ TEOA in MilliQ $\mathrm{H}_{2} \mathrm{O}$ (Fig. 5a) and allowed to sit in the dark for $3 \mathrm{~h}$ to completely soak in the sacrificial reductant. Aliquots $(500 \mu \mathrm{L})$ were collected during this initial $3 \mathrm{~h}$ period to track by UV-Vis spectrophotometry (Figs. S15-17) the quantity of MO released as the microparticles sat in the dark. After the initial induction period, the microparticles were irradiated with blue light (450 $\mathrm{nm}$ ) over several days (Fig. 5a) and the MO released was tracked once again by UV-Vis (Figs. S28, S32). A calibration curve (Fig S27) was constructed to facilitate conversion of UV-Vis absorbance to concentration of released $\mathrm{MO}$, and the corresponding percent release was calculated by comparing to the theoretical MO capacity. During the photoirradiation experiments, MO release was observed for three of four samples (Fig. 5b). The no-oligoviologen control microparticles (MP-4), crosslinked by PEGDA, was the only sample that displayed no MO release (Figs. S24-26 and S31, S35). This result was not surprising considering the excess $\mathrm{MO}$ dye that was added to these microparticles was easily washed away prior to the controlled release experiments. In other words, there were no viologen-based electrostatic loading sites present in the microparticle, so the molecular anion could be removed readily. For the no-irradiation control (MP-1_No-hv), we observed slow release of the dye up to 35\% after 5 d (Figs. S18-20 and S29, S33). We hypothesize this release is caused by the basic TEOA generating hydroxide $\left(\mathrm{OH}^{-}\right)$anions in $\mathrm{H}_{2} \mathrm{O}$, which could engage in counteranion exchange with the loaded $\mathrm{MO}$ dye anions. To test this hypothesis, an MP-1_No-hv batch was assessed in the dark, but in $0.3 \mathrm{mM}$ TEOA instead of $3 \mathrm{mM}$. The results from this control experiment (Fig. S36) demonstrated a lower release of
MO relative to experiments run with $3.0 \mathrm{mM}$ TEOA present. In the interest of time though, all microparticle release experiments were carried out in $3.0 \mathrm{mM}$ TEOA because the MO release was faster at this concentration. For the nophotocatalyst control microparticles (MP-3), 38\% of loaded MO was released over $5 \mathrm{~d}$ (Figs. S21-23, S30, S34), which is much less than the amount released from MP-1 (Fig 5b). We hypothesize that in addition to counteranion exchange, the irradiated oligoviologen crosslinker may experience some PET reduction of the viologen subunits in the presence of the styrene benzyl group, causing additional release. ${ }^{36}$ However, by far, the optimal performance of the photoredox-based microparticle platform was achieved when all components were present and irradiated (MP-1), thus following a PET-based reduction of viologens that yielded the fastest release. For example, more dye was released from irradiated MP-1 in $2 \mathrm{~d}$ than any control batch released over $5 \mathrm{~d}$. With the faster rate of release confirmed for MP-1, it was initially surprising to see the level of $\mathrm{MO}$ released plateaued at $60 \%$ after $5 \mathrm{~d}$. We speculated this upper limit could be due to osmotic pressure that limited release once more dye existed on the outside of the dialysis tubing than what remained inside the microparticles/tubing. To confirm this suspicion, once the percent release of $\mathrm{MO}$ plateaued at $\sim 60 \%$, the microparticles and the dialysis tubing they resided in were placed in a beaker containing fresh $3 \mathrm{mM}$ TEOA solution in $\mathrm{H}_{2} \mathrm{O}$. Continued irradiation with blue light of these microparticles resulted in additional release of the remaining $\mathrm{MO}$ up to $95 \%$ of the theoretical loading capacity (Fig. S37).

\section{Conclusion}

The design, synthesis, characterization, and electrostatic loading and controlled, visible light-induced release of dye from photoresponsive viologen-based microparticles is reported. The microparticles were synthesized through a W/O emulsion polymerization of aqueous, crosslinker-laden droplets emulsified within oil and supported by PGPR emulsifier. An oligoviologen dimer 
crosslinker and a PEGDA co-crosslinker were combined in the presence of an integrated porphyrin photocatalyst, enabling reduction of the resultant microparticle through visible-light photoredox catalysis. Methyl orange (MO), an anionic dye molecule, was successfully electrostatically loaded onto the small-molecule oligoviologen crosslinker in solution and into the microparticle postpolymerization through a simple counteranion metathesis exchange. The as-synthesized and dye-loaded microparticles were characterized by optical absorption and scanning electron microscopy, revealing a corona morphology within each individual microparticle. Using SEM, it was determined the as-synthesized microparticles measured $481 \pm 20.9 \mathrm{~nm}$ in diameter and the dyeloaded microparticles measured $785 \pm 18.2 \mathrm{~nm}$. DLS measurements showed that microparticles shrank briefly upon reduction but quickly swelled back to their initial size, presumably in response to counteranion metathesis and exposure to ambient $\mathrm{O}_{2}$. MO-loaded microparticles were irradiated in the presence of TEOA to demonstrate electrostatic release. UV-Vis spectrophotometry was used to track the release of MO over the course of $5 \mathrm{~d}$, followed by converting it to percent release and plotting the data. Control experiments revealed the critical importance of the viologen subunit as the loading and release site. When removing the photocatalyst, or not using visible light to activate the microparticles, the dye release was slowed and only reached a maximum of $38 \% \mathrm{MO}$ released over $5 \mathrm{~d}$. In the PET-reduced MP-1, however, we observed that the viologen-based photoreduction mechanism outcompetes simple counteranion exchange and yields faster release (reaching $40 \%$ released $\mathrm{MO}$ in just over $2 \mathrm{~d}$ and plateauing at $60 \%$ after $5 \mathrm{~d}$ ). The microparticle platform reported here serves as a promising proof-ofconcept for effective loading and temporal release of negatively charged functional cargo from small microparticles.

\section{Conflicts of interest}

The authors declare no conflict of interest.

\section{ORCID}

Mark S. Palmquist: 0000-0001-8158-5001

Max C. Gruschka:0000-0001-8769-4057

Jovelt M. Dorsainvil: 0000-0002-1770-4245

Abigail O. Delawder: 0000-0002-2297-9817

Tiana M. Saak: 0000-0001-6539-4205

Mary K. Danielson: 0000-0002-8542-6777

Jonathan C. Barnes: 0000-0003-2945-8691

\section{Author Contributions}

J.C.B. conceived the idea, and J.C.B. and M.S.P. designed the experiments. M.S.P., M.C.G., J.M.D., A.O.D., T.M.S., and M.K.D. synthesized and characterized all compounds and materials. J.C.B. and M.S.P. cowrote the manuscript and all authors contributed to the refinement of the manuscript and supporting information files.

\section{Acknowledgements}

We thank the David and Lucile Packard Foundation for J.C.B.'s Packard Fellowship for Science and Engineering which supported this research. M.S.P. acknowledges support from the Department of Defense (DoD) through the National Defense Science \& Engineering Graduate (NDSEG) Fellowship program. A.O.D. acknowledges support from the National Science Foundation Graduate Research Fellowship Program (NSF GRFP; DGE-1745038) and PEO International Scholar Award. T.M.S. acknowledges support from the American Chemical Society Division of Organic Chemistry's Summer Undergraduate Research Fellowship program. The mass spectrometry data was obtained using the NIH/NIGMS Biomedical Mass Spectrometry Resource at WUSTL, which was supported by a grant from the National Institutes of Health/National Institute of General Medical Sciences (\#8P41GM103422).

\section{Notes and references}

1. V. Subramanian, Microspheres: Technologies and Global Markets, 2018

2. O. Franssen and W. E. Hennink, Int J Pharmaceut, 1998 168, 1-7.

3. Y. J. Xia and D. W. Pack, Chem Eng Sci, 2015, 125, 129-143.

4. J. Kozlowska, W. Prus and N. Stachowiak, Int J Biol Macromol, 2019, 129, 952-956.

5. K. M. Z. Hossain, U. Patel and I. Ahmed, Prog Biomater, 2015, 4, 1-19.

6. K. Parida, S. Panda, P. Ravanan, H. Roy, M. Manickam and P. Talwar, Cellulose, 2013, 17, 18.

7. C. Y. Wong, H. Al-Salami and C. R. Dass, Int J Pharm, 2018, 537, 223-244.

8. S. R. White, N. R. Sottos, P. H. Geubelle, J. S. Moore, M. R. Kessler, S. R. Sriram, E. N. Brown and S. Viswanathan, Nature, 2001, 409, 794-797.

9. A. P. Esser-Kahn, N. R. Sottos, S. R. White and J. S. Moore, J Am Chem Soc, 2010, 132, 10266-10268.

10. A. P. Esser-Kahn, S. A. Odom, N. R. Sottos, S. R. White and J. S. Moore, Macromolecules, 2011, 44, 5539-5553.

11. E. Campos, J. Branquinho, A. S. Carreira, A. Carvalho, P. Coimbra, P. Ferreira and M. H. Gil, Eur Polym J, 2013, 49, 2005-2021.

12. C. M. Pitsillides, E. K. Joe, X. Wei, R. R. Anderson and C. P. Lin, Biophys J, 2003, 84, 4023-4032.

13. I. Ortiz de Solorzano, G. Mendoza, M. Arruebo and V. Sebastian, Colloids Surf B Biointerfaces, 2020, 190, 110904.

14. N. Vilanova, I. Feijter, A. J. P. Teunissen and I. K. Voets, Sci Rep, 2018, 8, 1271.

15. L. Michaelis and E. S. Hill, J Gen Physiol, 1933, 16, 859-873.

16. L. Striepe and T. Baumgartner, Chemistry, 2017, 23, 1692416940.

17. A. F. Greene, M. K. Danielson, A. O. Delawder, K. P. Liles, X. S. Li, A. Natraj, A. Wellen and J. C. Barnes, Chem Mater, 2017, 29, 9498-9508.

18. K. P. Liles, A. F. Greene, M. K. Danielson, N. D. Colley, A Wellen, J. M. Fisher and J. C. Barnes, Macromol Rapid Commun, 2018, 39, e1700781.

19. A. O. Delawder, A. Natraj, N. D. Colley, T. Saak, A. F. Greene and J. C. Barnes, Supramol Chem, 2019, 31, 523-531. 
20. F. Amir, K. P. Liles, A. O. Delawder, N. D. Colley, M. S. Palmquist, H. R. Linder, S. A. Sell and J. C. Barnes, ACS Appl Mater Interfaces, 2019, 11, 24627-24638.

21. F. Amir, X. Li, M. C. Gruschka, N. D. Colley, L. Li, R. Li, H. R. Linder, S. A. Sell and J. C. Barnes, Chem Sci, 2020, 11, 10910-10920.

22. J. J. Ding, C. N. Zheng, L. X. Wang, C. B. Lu, B. Zhang, Y. Chen, M. Q. Li, G. Q. Zhai and X. D. Zhuang, J Mater Chem A, 2019, 7, 23337-23360.

23. M. R. Geraskina, A. S. Dutton, M. J. Juetten, S. A. Wood and A. H. Winter, Angew Chem Int Ed Engl, 2017, 56, 94359439.

24. Q. Sui, X. T. Ren, Y. X. Dai, K. Wang, W. T. Li, T. Gong, J. J. Fang, B. Zou, E. Q. Gao and L. Wang, Chem Sci, 2017, 8, 2758-2768.

25. T. Janoschka, N. Martin, U. Martin, C. Friebe, S. Morgenstern, H. Hiller, M. D. Hager and U. S. Schubert, Nature, 2015, 527, 78-81.

26. C. L. Bird and A. T. Kuhn, Chem Soc Rev, 1981, 10, 49-82.

27. W. Griffin, Croda Oil \& Gas, 2014, DOI: 10.1002/jsfa.6444, 1-19.

28. M. Nazari, M. A. Mehrnia, H. Jooyandeh and H. Barzegar, J Food Process Eng, 2019, 42, 1-8.

29. F. Wolf, K. Koehler and H. P. Schuchmann, J Food Process Eng, 2013, 36, 276-283.

30. F. Y. Ushikubo and R. L. Cunha, Food Hydrocolloid, 2014, 34, 145-153.

31. R. Wilson, B. J. Van Schie and D. Howes, Food Chem Toxicol, 1998, 36, 711-718.

32. X. Schultze, J. Serin, A. Adronov and J. M. J. Frechet, Chem Commun, 2001, 1, 1160-1161.

33. C. E. McCusker and J. K. McCusker, Inorg Chem, 2011, 50, 1656-1669.

34. Q. Zhang, J. Dong, Q. Meng, G. Huang and S. Li, Russ J Gen Chem+, 2018, 88, 2388-2393.

35. N. Armaroli, G. Accorsi, D. Felder and J. F. Nierengarten, Chem-Eur J, 2002, 8, 2314-2323.

36. A. Mills, M. McFarlane and S. Schneider, Anal Bioanal Chem, 2006, 386, 299-305. 